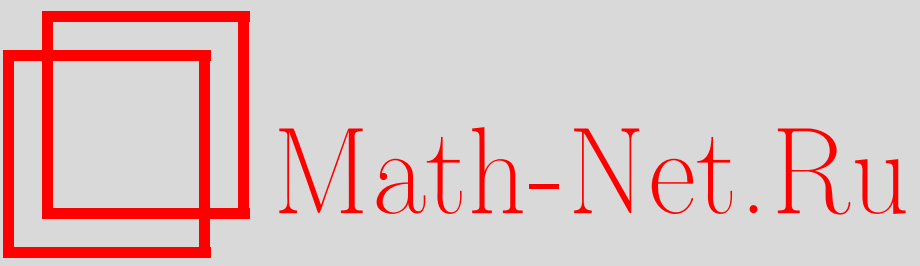

М. В. Ружанский, Об особенностях аффинных слоений некоторых типов, УМН, 2000, том 55, выпуск 2, 149-150

DOI: https://doi.org/10.4213/rm282

Использование Общероссийского математического портала Math-Net.Ru подразумевает, что вы прочитали и согласны с пользовательским соглашением

http://www.mathnet.ru/rus/agreement

Параметры загрузки:

IP: 54.166 .219 .16

26 апреля 2023 г., 17:09:40 


\title{
ОБ ОСОБЕННОСТЯХ АФФИННЫХ СЛОЕНИЙ НЕКОТОРЫХ ТИПОВ
}

\author{
М.В. РУжАнСкиЙ
}

В настоящей статье приводятся результаты об особенностях слоений, задаваемых ядрами голоморфных матричнозначных функций, обобщающие результаты [1]. Пусть $\Omega$ - связное открытое множество в $\mathbb{C}^{n}$. Рассмотрим голоморфное отображение $A: \Omega \rightarrow \mathbb{C}^{p \times n}, n, p \in \mathbb{N}$. Пусть $k=\max _{z \in \Omega} \operatorname{rank} A(z)$, и предположим, что $k \leqslant n-1$. Через $\Omega^{(k)}$ обозначим множество, на котором максимальньй ранг $k$ достигается, $\Omega^{(k)}$ открыто и плотно в $\Omega$. Для каждого $z \in \Omega^{(k)}$ ядро матрицы $A(z)$ имеет размерность $n-k$. Таким образом, получаем отображение $\kappa$ из $\Omega^{(k)}$ в $\mathbb{G}_{n-k}\left(\mathbb{C}^{n}\right)$, заданное формулой $\kappa(z)=\operatorname{ker} A(z)$. По теореме о неявной функции отображение $\kappa$ регулярно в $\Omega^{(k)}$. Одной из основных возникаюших проблем является нахождение условий голоморфной продолжимости $\kappa$ в область $\Omega$. В случае, когда такого продолжения не существует, обозначим через $\Omega^{\text {sing }}$ множество существенных особенностей $\kappa$. Оказывается возможным связать свойство продолжимости с размерностью множества $\Omega^{\text {sing }}$. Мы сосредоточимся на случае, когда $\kappa$ задает слоение в $\Omega$ в следуюшем смысле. В точке $z \in \Omega^{(k)}$ рассмотрим афинное подпространство $z+\kappa(z)$ в $\Omega$, проходящее через $z$. Для любого $w \in(z+\kappa(z)) \cap \Omega^{(k)}$ имеем афинное подпространство $w+\kappa(w)$, проходящее через $w$. Если предположить, что эти абфинные подпространства совпадают, т.е. $\kappa(z)=\kappa(w)$ для всех $w \in(z+\kappa(z)) \cap \Omega^{(k)}$, получаем локальное представление $\Omega^{(k)}$ как объединение непересекающихся афинных подпространств размерности $n-k$. Очевидно, пространства $z+\kappa(z)$ и $w+\kappa(w)$ локально в $\Omega^{(k)}$ либо совпадают, либо не пересекаются. Действительн, если бы они были различны, но пересекались бы в точке множества $\Omega^{(k)}$, то эта точка пересечения была бы также в $\Omega^{\operatorname{sing}}$, что невозможно, так как множества $\Omega^{(k)}$ и $\Omega^{\operatorname{sing}}$ не пересекаются. По другому условие слоения можно охарактеризовать как требование постоянства $\kappa$ вдоль $(z+\kappa(z)) \cap \Omega^{(k)}$. С этого момента будем полагать, что это условие выполнено, т.е. что локально $\kappa$ задает слоение афинными $(n-k)$-мерными подпространствами в $\Omega^{(k)}$.

ТЕОрема 1. Предположим, что множество существенных особенностей $\Omega^{\operatorname{sing}}$ не пусто. Тогда $\Omega^{\text {sing }}$ аналитично в $\Omega$ идля любого $\xi \in \Omega^{\text {sing }}$ выполнено $k-1 \leqslant \operatorname{dim}_{\xi} \Omega^{\text {sing }} \leqslant$ $n-2$.

Возникает важная ситуация, когда $A$ - якобиан голоморфного отображения $\Gamma: \Omega \rightarrow \mathbb{C}^{p}$. В этом случае условие, что $\kappa$ задает слоение, означает, что $\Gamma$ постоянна вдоль $z+\kappa(z)$ для любого $z \in \Omega^{(k)}$. Таким образом, поверхность уровня $\Gamma^{-1}(\Gamma(z))$ локально совпадает с $z+\kappa(z)$ для всех $z \in \Omega^{(k)}$. Можно показать, что они совпадают также глобально в $\Omega^{(k)}$. Слоения подобного типа возникают в следующей ситуации. Пусть $X$ - гладкое многообразие, и пусть $\Lambda$ коническое Лагранжево подмногообразие в $T^{*} X$. Через $\pi$ обозначим стандартную проекцию из $T^{*} X$ в $X$. Пусть $\Sigma=\pi(\Lambda), \Sigma_{0} \subset \Sigma$ - регулярное подмножество и $N^{*} \Sigma_{0}$ - конормальное расслоение к $\Sigma_{0}$. Обозначим через $\Phi$ производящую функцию для $\Lambda$, т.е. введем локальную параметризацию для $\Lambda$ в форме $\{(\nabla \Phi(\xi), \xi)\}$ [2]. Таким образом, сужение проекции $\pi$ на $\Lambda$ задается отображением $\nabla \Phi$. При естественном предположении, что $X$ и $\Lambda$ вещественно аналитичны (как в [3]), наше отображение $\Gamma$ есть не что иное, как аналитическое продолжение $\nabla \phi$ в комплексную область, и $A=\phi_{\xi \xi}^{\prime \prime}$, где $\phi$ получается из $\Phi$ путем факторизации конической переменной, например, если положить $\phi(\eta)=\Phi(\eta, 1)$ в некоторой канонической окрестности точки $\xi_{n+1}=1$ (где имеем также $n=\operatorname{dim} X-1$ ). Отметим, что размерность множества $\Omega^{\operatorname{sing}}$ может играть важную роль в проблемах регулярности интегральных операторов Фурье [1], [4], [5], так как $\Lambda=W F^{\prime}(F) \subset T^{*}(X \times Y)$ соответствует волновому фронту интегрального оператора Фурье $F$ из $Y$ в $X$.

Теорема 2. Пусть $A=D \Gamma \in \mathbb{C}^{p \times n}$ для голоморфного отображения $\Gamma: \Omega \rightarrow \mathbb{C}^{p}$. Предположим, что $\Omega^{\text {sing }}$ не пусто. Тогда в дополнение к оченкам теоремы 1 имеем $n-k+1 \leqslant \operatorname{dim}_{\xi} \Omega^{\text {sing }} \forall \xi \in \Omega^{\text {sing }}$. В частности, $3 \leqslant k \leqslant n-1 u n \geqslant 4$. Кроме того, 
пусть $\xi \in \Omega^{\text {sing }}$ - регулярная точка в $\Omega^{\operatorname{sing}}, \xi=\lim _{i \rightarrow \infty} \xi_{i}, \xi_{i} \in \Omega^{(k)}, u \mathbb{G}_{n-k}\left(\mathbb{C}^{n}\right) \ni$ $\kappa=\lim _{i \rightarrow \infty} \kappa\left(\xi_{i}\right)$. Тогда $\kappa \subset T_{\xi} \Omega^{\text {sing }}$.

В частности, можно заключить, что при $k \leqslant 2$ слоение, задаваемое $\kappa$, голоморфно продолжимо с $\Omega^{(k)}$ на все $\Omega$. Обратное к теореме 2 утверждение выполняется на уровне сушествования голоморфного отображения $\Gamma: \Omega \rightarrow \mathbb{C}^{p}$ с $A=D \Gamma$ :

Теорема 3. Для любы $3 \leqslant k \leqslant n-1 u \max \{k-1, n-k+1\} \leqslant d \leqslant n-2$ существует голоморфное отображение $\Gamma: \mathbb{C}^{n} \rightarrow \mathbb{C}^{n}$ такое, что $\operatorname{dim} \Omega^{\operatorname{sing}}=d n р и A=D \Gamma$. Более того, можно вибрать $Г$ с $\Omega \backslash \Omega^{(k)}=\Omega^{\text {sing }}$.

Случай с $A=\phi_{\xi \xi}^{\prime \prime}$, упомянутый выше, подробно рассмотрен в [1]. В этом случае условие на слоение для ядер матрицы $A$ можно записать в терминах функции $\phi$. Функция, задающая слоение, удовлетворяет системе однородных дифференциальных уравнений в частных производных первого порядка с коэффициентами, выражающимися через функцию $\phi$. С другой стороны, при заданном слоении функция $\phi$, Гессиан $A$ которой задает это слоение, удовлетворяет системе однородных дифференциальных уравнений в частных производных второго порядка с коэффициентами, выражающимися через слоение. С этими системами связан ряд интересных задач (см. [1]).

Как следует из замечания после теоремы 2 , наименьшая размерность, при которой не все особенности устранимы, это $n=4$. Пример, где множество $\Omega^{\text {Sing }}$ не пусто, можно дать с помощью функции

$$
\phi\left(\xi_{1}, \xi_{2}, \xi_{3}, \xi_{4}\right)=\xi_{1} \xi_{2}^{l}+\left(\xi_{3}-\xi_{2} \xi_{4}\right)^{m}, \quad l, m \geqslant 2 .
$$

\section{СПИСОК ЛИТЕРАТУРЫ}

[1] Ружанский М. В. // УМН. 2000. Т. 55. № 1. С. 99-170. [2] Duistermaat J. J. Fourier Integral Operators. Basel: Birkhäuser, 1996. [3] Arnol'd V. I. Singularities of Caustics and Wave Fronts. Dordrecht: Kluwer, 1990. [4] Ruzhansky M. // Arch. Math. 1999. V. 72. № 1. P. 68-76. [5] Seeger A., Sogge C. D., Stein E. M. // Ann. of Math. 1991. V. 134. P. 231-251.

Эдинбургский университет

Принято редколлегией

E-mail: ruzh@maths.ed.ac.uk 26.01 .2000 